\title{
Experimental constraints on nonlinearities induced by two-photon effects in elastic and inelastic Rosenbluth separations
}

\author{
V. Tvaskis,,${ }^{1,2}$ J. Arrington, ${ }^{3}$ M. E. Christy, ${ }^{1}$ R. Ent, ${ }^{2}$ C. E. Keppel,,${ }^{1,2}$ Y. Liang, ${ }^{1,4}$ and G. Vittorini ${ }^{5}$ \\ ${ }^{1}$ Hampton University, Hampton, Virginia 23668, USA \\ ${ }^{2}$ Thomas Jefferson National Accelerator Facility, Newport News, Virginia 23602, USA \\ ${ }^{3}$ Argonne National Laboratory, Argonne, Illinois 60439, USA \\ ${ }^{4}$ American University, Washington, D.C. 20016, USA \\ ${ }^{5}$ Eckerd College, St. Petersburg, Florida 33711, USA \\ (Received 18 November 2005; published 28 February 2006)
}

\begin{abstract}
The effects of two-photon exchange corrections, suggested to explain the difference between measurements of the proton elastic electromagnetic form factors using the polarization transfer and Rosenbluth techniques, have been studied in elastic and inelastic scattering data. Such corrections could introduce $\varepsilon$-dependent nonlinearities in inelastic Rosenbluth separations, where $\varepsilon$ is the virtual photon polarization parameter. It is concluded that such nonlinear effects are consistent with zero for elastic, resonance, and deep-inelastic scattering for all $Q^{2}$ and $W^{2}$ values measured.
\end{abstract}

DOI: 10.1103/PhysRevC.73.025206

PACS number(s): 13.40.Gp, 25.30.Fj, 12.20.Fv

\section{INTRODUCTION}

Electron-proton $(e-p)$ scattering has proven to be a powerful tool in the investigation of the structure of the nucleon. This interaction is typically described as the exchange between the electron and the proton of a single virtual photon. Because the electron is a point-like particle, any structure observed in $e-p$ scattering must be related to the target structure. Moreover, the relatively small value of the electromagnetic coupling constant ensures that corrections to the one-photon exchange approximation should be relatively small. To further improve on the already impressive accuracy achieved in the analysis of electron scattering data, the contribution of two-photon exchange (TPE) effects in elastic e-p scattering are under theoretical investigation [1-7]. Two-photon exchange effects have garnered particular attention as of late due to their potential role in resolving the discrepancy between electromagnetic form factors measured through the Rosenbluth separation method [8-11] and a polarization transfer technique [12,13] (see Sec. III).

Data from elastic and inelastic scattering, both in the resonance and deep-inelastic regimes, are here studied in light of this concern. There is a newly expanded, substantial data set which enables in particular a search for nonlinearities caused by TPE effects. While dedicated measurements have been proposed for elastic data [14], and a model-dependent analysis of nonlinearity has been performed for elastic $e-p$ scattering [15], this work represents a first detailed, modelindependent study of nonlinear effects in elastic and inelastic $e-p$ scattering data.

\section{ROSENBLUTH SEPARATION TECHNIQUE}

The differential cross section for $e-p$ scattering can be expressed in the Born approximation in terms of absorption of longitudinal $\left(\sigma_{L}\right)$ and transverse $\left(\sigma_{T}\right)$ virtual photons as

$$
\frac{d^{2} \sigma}{d \Omega d E^{\prime}}=\Gamma\left[\sigma_{T}\left(W^{2}, Q^{2}\right)+\varepsilon \sigma_{L}\left(W^{2}, Q^{2}\right)\right]
$$

where $Q^{2}$ is the negative squared mass of the virtual photon, $W^{2}$ is the mass squared of the undetected system, and $\Gamma$ is the transverse virtual photon flux:

$$
\Gamma=\frac{\alpha K}{2 \pi^{2} Q^{2}} \frac{E^{\prime}}{E} \frac{1}{1-\varepsilon} .
$$

Here, $\alpha$ is the fine structure constant, $E$ and $E^{\prime}$ are the energy of the initial and scattered electron, respectively, and $K$ is

$$
K=\frac{2 M v-Q^{2}}{2 M}
$$

where $M$ is the mass of the proton and $v=E-E^{\prime}$. The variable $\varepsilon$ is the relative longitudinal virtual photon flux. Therefore $\varepsilon=0$ corresponds to a purely transverse photon polarization.

The Rosenbluth separation technique is used to separate the longitudinal and transverse components of the cross section. Here, Eq. (1) is written in the following form:

$$
\sigma_{r} \equiv \frac{1}{\Gamma} \frac{d^{2} \sigma}{d \Omega d E^{\prime}}=\sigma_{T}\left(W^{2}, Q^{2}\right)+\varepsilon \sigma_{L}\left(W^{2}, Q^{2}\right) .
$$

In the Born approximation, the left hand side, the reduced cross section, depends linearly on $\varepsilon$. To perform the Rosenbluth separation, data covering a range in $\varepsilon$ at fixed $\left(W^{2}, Q^{2}\right)$ values must be obtained. Any deviation from linearity must come from higher order terms that are not included in the standard radiative correction procedures.

\section{TWO METHODS OF FORM FACTORS MEASUREMENTS AND TWO DIFFERENT RESULTS}

For the case of elastic scattering, the Rosenbluth separation technique is used to extract the form factors $G_{E}$ and $G_{M}$, from the $\varepsilon$ dependence of a reduced elastic cross section $\sigma_{r}$ at fixed $Q^{2}$. For elastic scattering, we define the reduced cross section 
as

$$
\sigma_{r} \equiv \frac{d \sigma}{d \Omega} \frac{\varepsilon(1+\tau)}{\sigma_{\text {Mott }}}=\tau G_{M}^{2}\left(Q^{2}\right)+\varepsilon G_{E}^{2}\left(Q^{2}\right),
$$

where $\tau=Q^{2} / 4 M^{2}$.

At fixed $Q^{2}$, the form factors $G_{E}$ and $G_{M}$ can be extracted from a linear fit in $\varepsilon$ to the measured reduced cross sections. Such a Rosenbluth fit yields $\tau G_{M}^{2}$ as the intercept and $G_{E}^{2}$ as the slope. With increasing $Q^{2}$, the cross section is dominated by $\tau G_{M}^{2}$, while the relative contribution of the $G_{E}^{2}$ term is diminished. Precise understanding of the $\varepsilon$-dependence in the radiative corrections becomes crucial at high values of $Q^{2}$. Therefore, in order to measure the ratio $G_{E} / G_{M}$ at high values of $Q^{2}$, a polarization transfer method has also been employed in Hall A at Jefferson Lab (JLab).

In polarized elastic electron-proton scattering, the longitudinal and transverse components of the recoil polarization are sensitive to different combinations of the electric and magnetic elastic form factors $[16,17]$. The ratio of the form factors can be directly related to the components of the recoil polarization

$$
\frac{G_{E}}{G_{M}}=\frac{P_{t}}{P_{l}} \frac{\left(E+E^{\prime}\right) \tan \left(\theta_{e} / 2\right)}{2 M},
$$

where $P_{l}$ and $P_{t}$ are the longitudinal and transverse components of the final proton polarization, and $\theta_{e}$ is the angle between the initial and final directions of the lepton.

Recent measurements from Jefferson Lab using the polarization transfer technique to measure the ratio $G_{E} / G_{M}$ have found that $G_{E}$ decreases more rapidly than $G_{M}$ at large $Q^{2}$ [12,13]. This differs from results obtained at SLAC in a similar $Q^{2}$ range using the Rosenbluth technique. There exist but two explanations for this discrepancy. There is either an unidentified systematic experimental uncertainty in the polarization transfer data, or a systematic uncertainty common to all Rosenbluth data.

It has been estimated that a 5-7\% systematic correction to the $\varepsilon$ dependence of the reduced Rosenbluth cross section measurements would be needed in order to resolve the discrepancy [18-20]. However, a detailed analysis does not show any inconsistencies in the cross section data sets [9]. Moreover, new high $Q^{2}$ cross section data from Jefferson Lab $[10,11]$ are consistent with the older SLAC cross section data [21] obtained in the same $Q^{2}$ range. The results of Ref. [11], where the struck proton rather than the scattered electron was detected, have a precision comparable to the polarization transfer measurements. It must be concluded, then, that the needed 5-7\% $\varepsilon$-dependent correction is not due to standard experimental considerations in the measured Rosenbluth cross sections.

It has been suggested [2,19] that the discrepancy may be explained by TPE effects not fully accounted for in the standard radiative corrections procedure of Mo and Tsai [22]. The polarization transfer technique involves a ratio of cross sections, and hence is expected not to be very sensitive to such effects $[1,4,6]$. In contrast, these contributions can significantly affect the Rosenbluth separation technique. TPE contributions can be independent of $\varepsilon$ [affecting both $G_{E}^{2}$ and $G_{M}^{2}$ in Eq. (5)], linear in $\varepsilon$ (significantly affecting $G_{E}^{2}$ ), or nonlinear in $\varepsilon$.
The experimental evidence for significant TPE contributions to the form factor measurements is still quite limited. While the nonzero transverse beam spin asymmetry [23,24] provides direct evidence for TPE in elastic $e-p$ scattering, we do not have any evidence for the large changes in the unpolarized cross sections ( $25 \%$ ) necessary to explain the difference between polarization transfer and Rosenbluth separation measurements. The discrepancy between polarization transfer and Rosenbluth extractions of $G_{E} / G_{M}$ provides only an indirect indication of a missing correction, while direct searches for TPE through the comparison of electron-proton and positronproton scattering yield some evidence of deviations from the Born approximation at low $\varepsilon$, but only at the three sigma level [25]. Observation of a deviation from linearity in the reduced cross section would provide a clear indication of TPE (or other higher order corrections not included in standard radiative correction procedures), though only the nonlinear portion of the correction could be directly isolated. New highprecision Rosenbluth data in elastic [11] and inelastic [26] $e-p$ scattering allow for a much more sensitive search for such nonlinearities.

This work reports results of a search for effects of TPE corrections in elastic and inelastic scattering data by searching for $\varepsilon$-dependent nonlinearities in existing Rosenbluth separations. We note that this analysis will not be sensitive to either systematic shifts in the reduced cross section of Eq. (4), or to two-photon effects which are linear in $\varepsilon$.

\section{DATA OVERVIEW}

Table I lists the data sets included in the present analysis. We include several measurements of elastic $e-p$ to cover a range in $Q^{2}$, while the SLAC measurements [27,28] and the recent JLab measurement [26] cover the DIS and resonance regions.

For elastic $e-p$ scattering, we examine Rosenbluth extractions from several different experiments. We study the Rosenbluth separation for the experiments and $Q^{2}$ values

TABLE I. Summary of experiments included in the analysis, including the number of L-T separations and the typical cross section uncertainties (excluding normalization uncertainties).

\begin{tabular}{lcrrl}
\hline \hline Elastic data & $\begin{array}{c}Q^{2} \\
{\left[\mathrm{GeV}^{2}\right]}\end{array}$ & $\begin{array}{c}\text { \# of } \\
\text { L-Ts }\end{array}$ & $\begin{array}{c}\text { Typ. } \\
\delta \sigma / \sigma\end{array}$ & Lab \\
\hline Janssens et al. $[29]$ & $0.2-0.9$ & 20 & $4.7 \%$ & Mark III \\
Litt et al. [30] & $2.5-3.8$ & 4 & $1.7 \%$ & SLAC \\
Berger et al. $[31]$ & $0.4-1.8$ & 8 & $2.6 \%$ & Bonn \\
Walker et al. $[8]^{\text {a }}$ & $1.0-3.0$ & 4 & $1.1 \%$ & SLAC \\
Andivahis et al. [21] & $1.8-5.0$ & 5 & $1.3 \%$ & SLAC \\
Christy et al. $[10]$ & $0.9-5.2$ & 7 & $1.3 \%$ & JLab \\
Qattan et al. $[11]^{\mathrm{c}}$ & $2.6-4.1$ & 3 & $0.6 \%$ & JLab \\
Inelastic data & $W^{2}\left[\mathrm{GeV}^{2}\right]$ & & & \\
\hline Liang et al. $[26]$ & $1.3-3.9$ & 191 & $1.7 \%$ & JLab \\
Dasu et al. $[27,28]$ & $3.2-30$ & 61 & $3.0 \%$ & SLAC \\
\hline \hline
\end{tabular}

\footnotetext{
${ }^{\mathrm{a}}$ Data below $20^{\circ}$ are excluded.

${ }^{\mathrm{b}}$ Data from $8 \mathrm{GeV}$ spectrometer.

"Excludes "slope" systematic uncertainties.
} 
TABLE II. Extracted values and 95\% confidence level upper limit on $P_{2} . \Delta_{\max }$ is the upper limit on deviations of the cross section from linearity [Eq. (8)].

\begin{tabular}{lrrr}
\hline \hline & $\left\langle P_{2}\right\rangle$ & $\left|P_{2}\right|_{\max }$ & $\begin{array}{c}\Delta_{\max } \\
95 \% \text { C.L. }\end{array}$ \\
\hline Elastic & $0.019 \%$ C.L. \\
Resonance & $-0.060(42)$ & 0.064 & $0.8 \% \cdot(\Delta \varepsilon)^{2}$ \\
DIS & $-0.012(71)$ & 0.086 & $1.1 \% \cdot(\Delta \varepsilon)^{2}$ \\
\hline \hline
\end{tabular}

listed in Table II of Ref. [9], including the updated radiative corrections [9]. In addition, data from two recent Jefferson Lab measurements $[10,11]$ are included. In all cases, the reduced cross sections are taken from a single experiment and single detector. Where necessary, cross section values at slightly different $Q^{2}$ values are shifted to a fixed $Q^{2}$ values. Only small corrections were needed, typically below $2 \%$, although a handful of points were corrected by $5-10 \%$. There are a total of 51 Rosenbluth separations that we will examine for nonlinearities. Typical point-to-point uncertainties are roughly $1-2 \%$ for most of the data sets, although several of the older experiment had larger uncertainties and the E01-001 results [11] have point-to-point uncertainties below one percent.

For the resonance region, we used newly obtained data from JLab Hall C experiment E94-110 [26,32], which was utilized to separate the longitudinal and transverse unpolarized proton structure functions in the nucleon resonance region via the Rosenbluth separation technique. The experiment ran with seven different energies ranging from 1.2 to $5.5 \mathrm{GeV}$ over a scattering angle range $12.9<\theta_{e}<79.9$. The total pointto-point uncertainty on the cross section measurements was approximately $2 \%$ [32]. The data taken from this experiment were used to perform 191 Rosenbluth separations covering the kinematic region $0.5<Q^{2}<5.0 \mathrm{GeV}^{2}$ and $1.1<W^{2}$ $<4.0 \mathrm{GeV}^{2}$. Examples of these Rosenbluth separations are shown in Fig. 1. These data were used to extract the ratio, $R$, of longitudinal to transverse cross section components. Rosenbluth separations are performed in five $Q^{2}$ bins and $43 W^{2}$ bins, yielding separations for inclusive scattering over the entire resonance region. The cross section values are interpolated to the central $W^{2}$ and $Q^{2}$ values of each bin using a global fit to the world's resonance region data [26,33], with constraints built in to provide a smooth transition to the DIS region and the $Q^{2} \rightarrow 0$ limit. Typical corrections were $5 \%$, and data that required corrections larger than $60 \%$ were excluded. The uncertainty in the interpolation was generally much smaller than the experimental uncertainties and was neglected in the analysis. While a few points had large enough corrections that the uncertainty in the interpolation may not be negligible for the given data set, their effect on the extracted values of any nonlinear terms should be small and random, thus largely canceling and providing a negligible contribution to the uncertainty in the extracted limits on nonlinearity.

For the deep-inelastic scattering (DIS) region, the data from experiment E140 at SLAC [27,28] have been used. A total of 61 Rosenbluth separations have been performed covering the kinematic region $0.63<Q^{2}<20 \mathrm{GeV}^{2}$ and $2.5<W^{2}<$

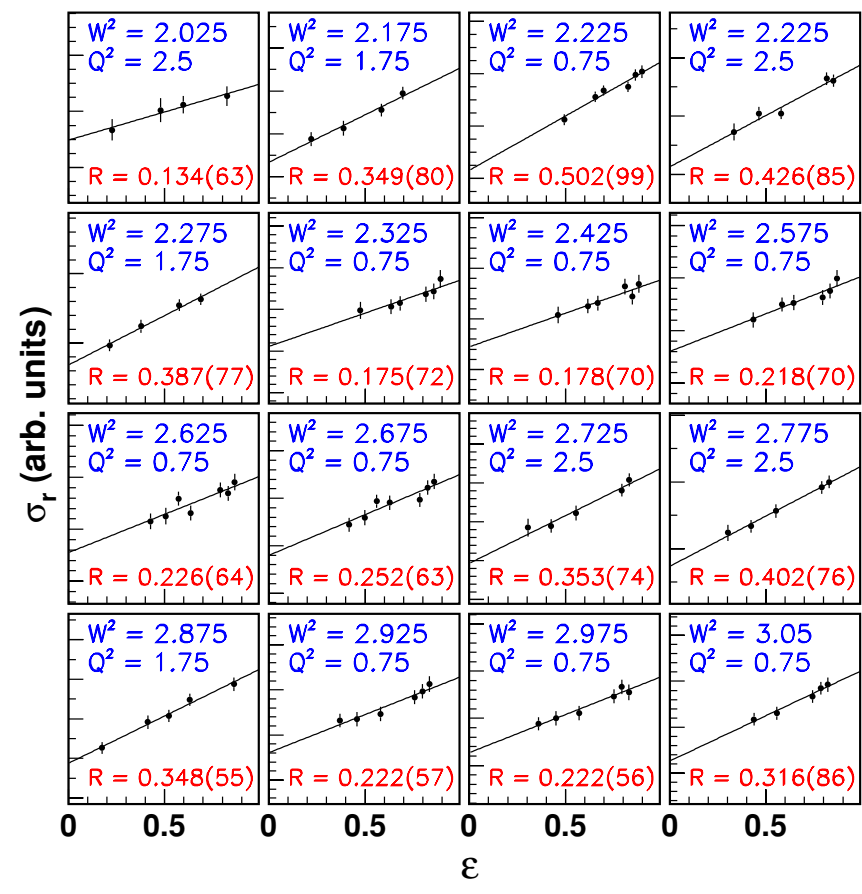

FIG. 1. (Color online) Example Rosenbluth separations performed in JLab experiment E94-110 [26]. Each panel shows the reduced cross section [Eq. (4)] vs $\varepsilon$ at a fixed value of $W^{2}$ and $Q^{2}$ (in $\mathrm{GeV}^{2}$ ), and the extracted value of $R=\sigma_{L} / \sigma_{T}$.

$30 \mathrm{GeV}^{2}$. The total point-to-point uncertainties on the cross section measurements depends on the actual kinematics, but are typically $2-3 \%$. Example Rosenbluth separations from E140 are shown in Fig. 2.

The data from the JLab E94-110 and SLAC E140 experiments are by far the largest data sets available for inelastic Rosenbluth separations, while the new elastic measurements from JLab E01-001 provide significantly more precise data for elastic scattering.

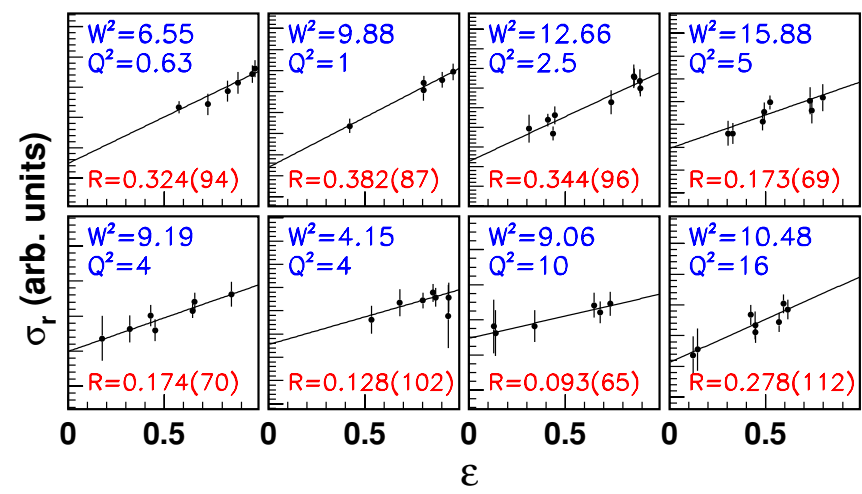

FIG. 2. (Color online) Example Rosenbluth separations performed in SLAC experiment E140 [27,28]. Each panel shows the reduced cross section [Eq. (4)] vs $\varepsilon$ at a fixed value of $W^{2}$ and $Q^{2}$ (in $\mathrm{GeV}^{2}$ ), and the extracted value of $R=\sigma_{L} / \sigma_{T}$. 


\section{ANALYSIS AND RESULTS}

From the discussions of Secs. II and III it is clear that the linearity of the reduced cross section in Eq. (4) is a crucial component of the Rosenbluth technique, and that two-photon exchange corrections could introduce a nonlinear $\varepsilon$-dependence in Eq. (4). Therefore, such corrections could manifest themselves as nonlinearities in Figs. 1 and 2.

To make a general, model-independent search for such nonlinearities, the following analysis has been performed. For each data set with three or more $\varepsilon$ values at fixed $Q^{2}$ and $W^{2}$, the reduced cross sections are fit to a quadratic in $\varepsilon$, of the form

$$
\sigma_{r}=P_{0} \cdot\left[1+P_{1}(\varepsilon-0.5)+P_{2}(\varepsilon-0.5)^{2}\right] .
$$

This form is chosen so that the extracted nonlinear term, $P_{2}$, will be a fractional contribution, relative to the average $(\varepsilon=0.5)$ cross section, rather than an absolute contribution to the cross section. One could also take the quadratic term relative to the $\varepsilon=0$ cross section, but this would greatly enhance the apparent size of the nonlinear contributions for data with $\sigma_{L} \ll \sigma_{T}$ and would lead to large uncertainties for data sets with only large $\varepsilon$ values, where there is a significant extrapolation to $\varepsilon=0$. In the absence of TPE, we expect $\sigma_{r}=\sigma_{T}+\varepsilon \sigma_{L}$, yielding $P_{0}=\sigma_{T}+0.5 \sigma_{L}, P_{0} P_{1}=\sigma_{L}$, and $P_{2}=0$. TPE corrections can modify $P_{0}$ and $P_{1}$, and may introduce a nonzero value of $P_{2}$. The only estimates we have for the size of the nonlinearity come from calculations for elastic $e-p$ scattering. If one takes the calculations [2,3] of TPE effects for elastic scattering and scales the size of the corrections so that they are large enough to explain the discrepancy between polarization and Rosenbluth extractions, as done in Ref. [14], one obtains $P_{2}$ values of $\approx 6-9 \%$, although the precise value depends significantly on $Q^{2}$ and the $\varepsilon$ range of the data.

While $P_{2}$ represents the fractional curvature, the size of cross section deviations from linearity will be much smaller. For $P_{2}=10 \%$, the maximum deviation of the cross section from $P_{2}=0$ would be $2.5 \%$, at $\varepsilon=0,1$. The effects are even smaller if the $\varepsilon$ range of the data, $\Delta \varepsilon$, is less than one. In this case, the deviations from $P_{2}=0$ will go approximately as $(\Delta \varepsilon)^{2}$. Finally, when one performs the Rosenbluth separation, the extracted values of $\sigma_{L}$ and $\sigma_{T}$ will be shifted from their true values in order to minimize the deviation from the straight line fit, reducing the deviations by roughly a factor of two from the size of the $P_{2}$ contribution in Eq. (7). Thus, the maximum observed deviations from linearity will be,

$$
\Delta_{\max }=\frac{\left(\sigma-\sigma_{\mathrm{fit}}\right)_{\max }}{\sigma} \approx P_{2} \cdot(\Delta \varepsilon)^{2} / 8,
$$

typically more than a factor of ten smaller than the value of $P_{2}$. For the expected $P_{2}$ values of $\lesssim 10 \%$ and a rather large $\Delta \varepsilon$ range of 0.8 , one expects maximum deviations from linearity to be at the level of $\lesssim 0.8 \%$. So even with high precision measurements and a good $\varepsilon$ range, one needs a large data set to provide meaningful limits on the nonlinearities.

We perform the fit from Eq. (7) for each of the elastic, resonance region, and DIS Rosenbluth data sets. Figures 3 and 4 show $P_{2}$, binned in $Q^{2}$ for the elastic data, and binned in $W$ for the resonance and DIS data. The results are consistent

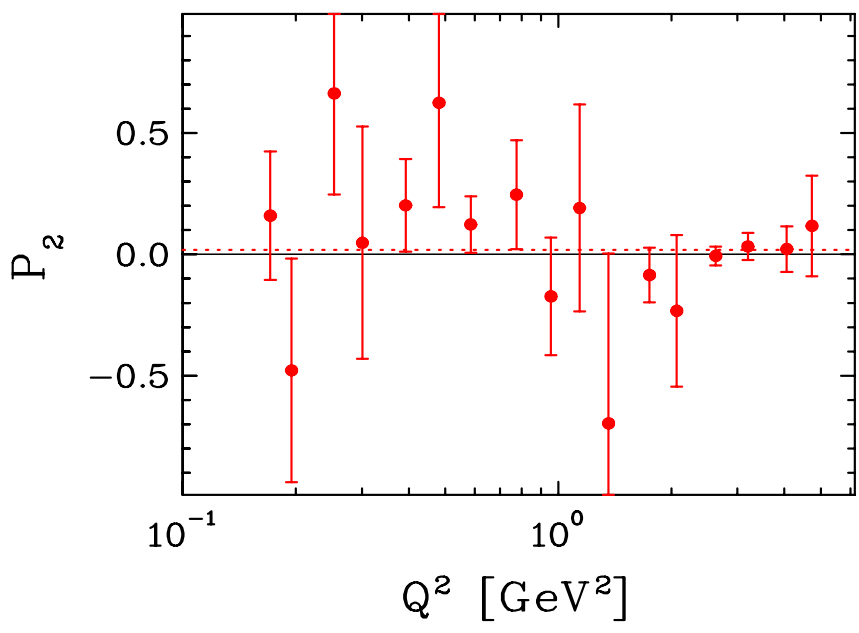

FIG. 3. (Color online) Extracted values of the curvature parameter, $P_{2}$, as extracted from the elastic data as a function of $Q^{2}$. The red dotted line indicates the average value, $\left\langle P_{2}\right\rangle=0.019 \pm 0.027$.

with no nonlinearities, and there is no apparent $Q^{2}$ or $W^{2}$ dependence. The best constraints for the elastic scattering come from the $Q^{2}=2.64,3.2 \mathrm{GeV}^{2}$ data from most recent Jefferson Lab measurement [11], which by themselves yield $\left\langle P_{2}\right\rangle=0.013(33)$, compared to previous worlds data which yields $\left\langle P_{2}\right\rangle=0.028(46)$. Table II shows the extracted value for $P_{2}$, the $95 \%$ confidence level upper limit on $\left|P_{2}\right|$, and the approximate maximum deviation from linearity for the elastic, resonance region, and DIS $\left(W^{2}>4 \mathrm{GeV}^{2}\right)$ data. From these results, we determine the $95 \%$ confidence level upper limits on $\left|P_{2}\right|$ of $6.4 \%$ for the elastic data and $10.7 \%$ for the inelastic data. This yields limits on the deviations of the data from the Rosenbluth fit of roughly $0.4 \%(0.7 \%)$ for the elastic (inelastic), assuming a $\Delta \varepsilon$ range of 0.7 .

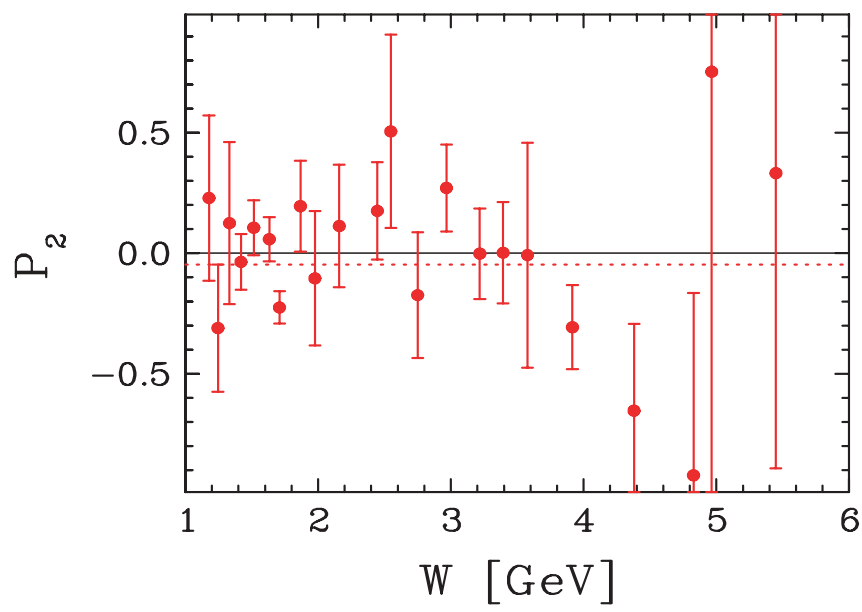

FIG. 4. (Color online) Extracted values of the curvature parameter, $P_{2}$, as extracted from the inelastic data as a function of $W$. Data in each $W$ bin is averaged over all $Q^{2}$ values in the resonance region and DIS measurements. The red dotted line indicates the average value, $\left\langle P_{2}\right\rangle=-0.048 \pm 0.036$. 
Note that it is also possible for a purely linear correction to introduce a small nonlinearity, since

$$
\frac{\sigma_{r}}{\sigma_{T}}=(1+R \varepsilon) \cdot\left(1+C_{2 \gamma} \varepsilon\right)=1+\left(R+C_{2 \gamma}\right) \varepsilon+R \cdot C_{2 \gamma} \varepsilon^{2},
$$

where $R=\sigma_{L} / \sigma_{T}$ and $\left(1+C_{2 \gamma} \varepsilon\right)$ is the TPE correction factor. For the elastic data at high $Q^{2}$ and all of the inelastic data presented here, $R \lesssim 0.2-0.3$, while estimates TPE predict a change in slope, $C_{2 \gamma}$, of approximately $5 \%$. Hence, the nonlinear term arising from a linear TPE correction, $R \cdot C_{2 \gamma}$, will be very small, yielding $P_{2} \lesssim 1 \%$. At low $Q^{2}$ values, the value of $R$ for the elastic cross section becomes quite large, yielding values of $P_{2}$ on the scale of $C_{2 \gamma}$ for $R \gtrsim 1$. However, $R$ is only this large for $Q^{2} \lesssim 0.4 \mathrm{GeV}^{2}$, where the TPE corrections decrease as $Q^{2} \rightarrow 0[6,19,20,25]$. The effect is $\lesssim 1 \%$ if one assumes that $C_{2 \gamma}$ increases slowly as one goes up from $Q^{2}=0$, as it does in calculations [6] and phenomenological extractions of the TPE corrections $[19,20]$. If one takes a more rapid increase with $Q^{2}, C_{2 \gamma}=0.06 \cdot\left[1-\exp \left(Q^{2} / 0.5 \mathrm{GeV}^{2}\right)\right]$, we obtain values of $P_{2}$ coming from the linear correction of $1.5-2.5 \%$ for $Q^{2}<1 \mathrm{GeV}^{2}$. Thus, the size of this effect should be well below the sensitivity of the existing data in all cases.

To better visualize the limits on nonlinearities, we have also performed a global comparison of the residuals between the reduced cross sections and a linear fit to the reduced cross sections. For the previous fit, data sets with a very small $\Delta \varepsilon$ range have very little sensitivity to the curvature. Although these data sets have large uncertainties, they still provide meaningful $P_{2}$ values. When plotting the residuals, we want to exclude such data sets because the data points have uncertainties comparable to the other data sets, but the residuals little sensitivity to nonlinearities. Thus, we require include only those data sets where $\Delta \varepsilon \geqslant 0.4$ when studying the residuals. This cut reduces the number of data sets to 35 for elastic kinematics, 77 in the resonance region, and 38 in the DIS region.

For the data sets with sufficient $\varepsilon$ coverage, we take $R_{1 \gamma}$ to be the residual from the Rosenbluth (one-photon exchange) fit,

$$
R_{1 \gamma}=\frac{\sigma_{\text {Data }}-\sigma_{\text {fit }}}{\sigma_{\text {fit }}}
$$

and obtain a value of $R_{1 \gamma}$ for every cross section measurement in the Rosenbluth data sets. We can then determine the weighted average value from the extracted $R_{1 \gamma}$ values in $\varepsilon$ bins for the elastic, resonance region, and inelastic data sets. In the absence of TPE contributions, one expects $R_{1 \gamma}=0$ in every $\varepsilon$ bin and, hence, any $\varepsilon$ dependence to $R_{1 \gamma}$ is an indication of two-photon exchange. Figure 5 shows the combined $R_{1 \gamma}$ as a function of $\varepsilon$ for the elastic, resonance region, and DIS data, and all three data sets are consistent with $R_{1 \gamma}=0$. One can see that while the elastic and resonance region data have high precision, the resonance region data has less data as $\varepsilon \rightarrow 0$ and 1 , while the DIS data has limited data an low $\varepsilon$, as well as lower statistical precision in general.

We fit the combined residuals to the form

$$
R_{1 \gamma}=A+B\left(\varepsilon-\varepsilon_{0}\right)^{2},
$$

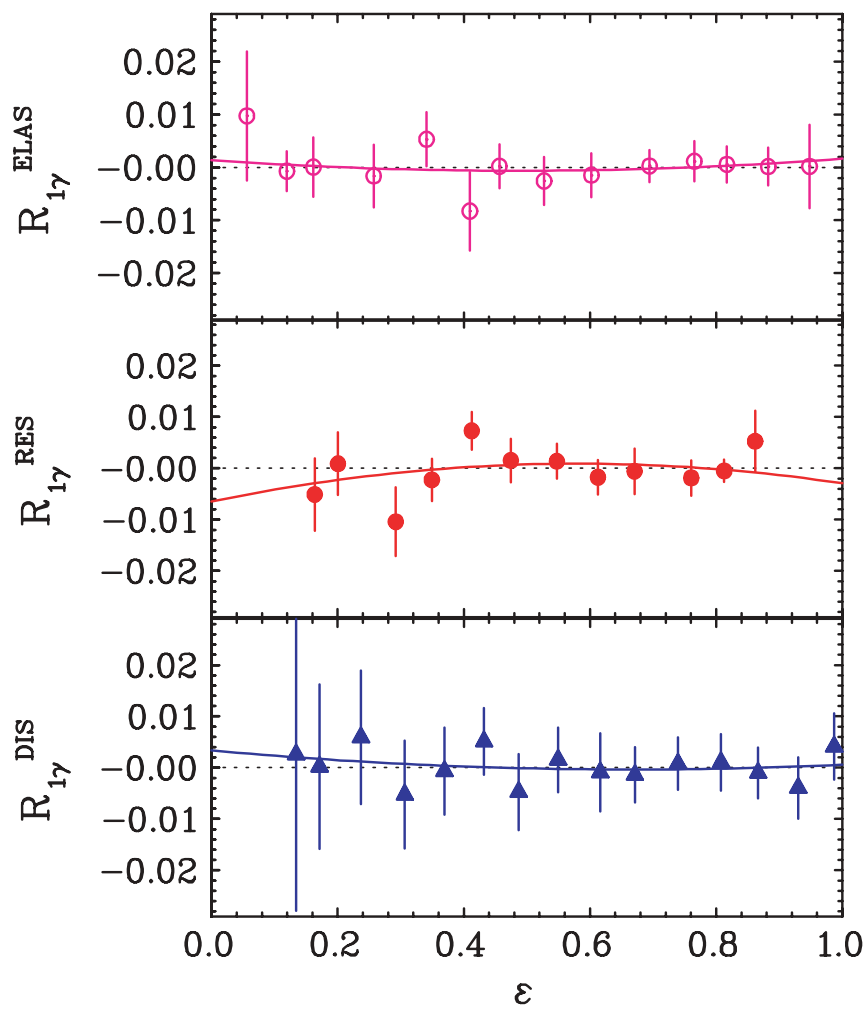

FIG. 5. (Color online) The weighted average of $R_{1 \gamma}=\left(\sigma_{\text {Data }}-\right.$ $\left.\sigma_{\text {Fit }}\right) / \sigma_{\text {Fit }}$ for the elastic measurements, the resonance region data (JLab experiment E94-110) and the DIS data (SLAC experiment E140). The solid lines are the fits to the form of Eq. (11).

where $A, B$, and $\varepsilon_{0}$ are the fit parameters. Because we are fitting to residuals that have already had the expected linear cross section dependence removed, we expect that $R_{1 \gamma}$ will average to zero, yielding $A \approx 0$ in the absence of any strong $\varepsilon$ dependence. Indeed, we find $A \lesssim 0.05 \%$ for the elastic, resonance, and DIS data. The quadratic fit to $R_{1 \gamma}$ yields a curvature parameter, $B$, consistent with zero for all data sets. We obtain $B=(0.9 \pm 2.0) \%$ for the elastic data, $(-2.3 \pm$ $3.0) \%$ for the resonance region data, and $(0.9 \pm 3.8) \%$ for the DIS measurements.

While the limits in Table II provide the best quantitative limits on deviations from linearity, the residuals shown in Fig. 5 give a better idea of the sensitivity of the different data sets in different regions of $\varepsilon$. The parametrization of Eq. (7) assumes a simple quadratic nonlinear term, while some models for the contribution to elastic scattering indicate larger nonlinearities for $\varepsilon \rightarrow 1$. From Fig. 5 we see that this region is not as well constrained for the resonance region data, while very low $\varepsilon$ values are not well constrained except in the elastic data.

\section{CONCLUSION}

We have searched for possible two-photon exchange contributions that show up as nonlinearities in Rosenbluth separations. We have used existing data in the elastic and 
deep-inelastic scattering region and recent data in the nucleon resonance region. We do not find any evidence for TPE effects. The $95 \%$ confidence level upper limit on the curvature parameter, $P_{2}$, was found to be $6.4 \%(10.6 \%)$ for the elastic (inelastic) data. This limits maximum deviations from a linear fit to $\lesssim 0.4 \%(0.7 \%)$ for typical elastic (inelastic) Rosenbluth separation data sets.

\section{ACKNOWLEDGMENTS}

The authors wish to thank Arie Bodek for useful discussions and comments. This work was supported in part by research grants 0099540 and 9633750 from the National Science Foundation and DOE grant W-31-109ENG-38.
[1] L. C. Maximon and W. C. Parke, Phys. Rev. C 61, 045502 (2000).

[2] P. G. Blunden, W. Melnitchouk, and J. A. Tjon, Phys. Rev. Lett. 91, 142304 (2003).

[3] Y. C. Chen, A. Afanasev, S. J. Brodsky, C. E. Carlson, and M. Vanderhaeghen, Phys. Rev. Lett. 93, 122301 (2004).

[4] A. V. Afanasev, S. J. Brodsky, C. E. Carlson, Y.-C. Chen, and M. Vanderhaeghen, Phys. Rev. D 72, 013008 (2005).

[5] M. P. Rekalo and E. Tomasi-Gustafsson, Eur. Phys. J. A 22, 331 (2004).

[6] P. G. Blunden, W. Melnitchouk, and J. A. Tjon, Phys. Rev. C 72, 034612 (2005).

[7] S. Kondratyuk, P. G. Blunden, W. Melnitchouk, and J. A. Tjon, Phys. Rev. Lett. 95, 172503 (2005).

[8] R. C. Walker et al., Phys. Rev. D 49, 5671 (1994).

[9] J. Arrington, Phys. Rev. C 68, 034325 (2003).

[10] M. E. Christy et al., Phys. Rev. C 70, 015206 (2004).

[11] I. A. Qattan et al., Phys. Rev. Lett. 94, 142301 (2005).

[12] O. Gayou et al., Phys. Rev. Lett. 88, 092301 (2002).

[13] V. Punjabi et al., Phys. Rev. C 71, 055202 (2005).

[14] J. Arrington et al., Jefferson lab experiment E05-017.

[15] E. Tomasi-Gustafsson and G. I. Gakh, Phys. Rev. C 72, 015209 (2005).
[16] A. I. Akhiezer and M. P. Rekalo, Sov. J. Part. Nuclei 4, 277 (1974).

[17] R. G. Arnold, C. E. Carlson, and F. Gross, Phys. Rev. C 23, 363 (1981).

[18] J. Arrington, Phys. Rev. C 69, 022201(R) (2004).

[19] P. A. M. Guichon and M. Vanderhaeghen, Phys. Rev. Lett. 91, 142303 (2003).

[20] J. Arrington, Phys. Rev. C 71, 015202 (2005).

[21] L. Andivahis et al., Phys. Rev. D 50, 5491 (1994).

[22] L. W. Mo and Y.-S. Tsai, Rev. Mod. Phys. 41, 205 (1969).

[23] S. P. Wells et al. (SAMPLE), Phys. Rev. C 63, 064001 (2001).

[24] F. E. Maas et al., Phys. Rev. Lett. 94, 082001 (2005).

[25] J. Arrington, Phys. Rev. C 69, 032201(R) (2004).

[26] Y. Liang et al. (Jefferson Lab Hall C E94-110), nucl-ex/0410027.

[27] S. Dasu et al., Phys. Rev. Lett. 61, 1061 (1988).

[28] S. Dasu et al., Phys. Rev. D 49, 5641 (1994).

[29] T. Janssens, R. Hofstadter, E. B. Huges, and M. R. Yearian, Phys. Rev. 142, 922 (1966).

[30] J. Litt et al., Phys. Lett. B31, 40 (1970).

[31] C. Berger, V. Burkert, G. Knop, B. Langenbeck, and K. Rith, Phys. Lett. B35, 87 (1971).

[32] Y. Liang, Ph.D. thesis, American University (2002).

[33] I. Niculescu et al., Phys. Rev. Lett. 85, 1186 (2000). 